\title{
ALGORITHM FOR SOLVING THE LIGHT SCATTERING PROBLEM FOR ARBITRARY SHAPED ICE PARTICLES WITH ABSORPTION
}

\author{
Timofeev D.N., Konoshonkin A.V., Kustova N.V., Borovoi A.G.
}

\author{
V.E. Zuev Institute of Atmospheric Optics, Rus. Acad. Sci., Tomsk, 1 Academician Zuev Sq. 634055, \\ Russia \\ *Email: tdn@iao.ru
}

\begin{abstract}
The work presents the estimation of the absorption influence effect on the light scattering problem for atmospheric ice particles. The calculation of light scattering matrices was performed for two types of particles: the solid hexagonal columns and the arbitrary shaped particles. The range of the size for both types of particles varies from $10 \mu \mathrm{m}$ to $1000 \mu \mathrm{m}$. The results of the research show an insignificant decrease of the value of the M11 element of the light scattering matrix for small-sized particles for all chosen wavelengths $(0.355 \mu \mathrm{m}, 0.532 \mu \mathrm{m}$, $1.064 \mu \mathrm{m}$ и $1.6 \mu \mathrm{m}$ ), and a significant decrease for big-sized particles for a $1.6 \mu \mathrm{m}$ wavelength.
\end{abstract}

\section{INTRODUCTION}

The light scattering problem of ice crystal particles in cirrus clouds is crucial for investigation of the solar energy transfer process. Cirrus clouds are an essential part of all clouds that covers Earth's surface because of this the recovering of their optical and microphysical properties is required to refine global climate models [1].

Cirrus clouds consist of ice crystals particles with various shape and size. For solving the light scattering problem for ice particles it is difficult to apply exact numerical methods such as the finite difference time domain method (FTDT) [2, 3] or the discrete dipole approximation method (DDA) $[4,5]$ due to their high requirements for computational resources for big-sized particles [6]. For this reason the problem is often solved with the geometrical and the physical optics approximation [7].

During the numerical calculation of light scattering matrices for ice particles it is important to take the specific factors that could influence on scattering into account. One of these factors is the absorption of light in ice particles.
The one of the important properties of an absorptive medium is refractive index that is complex and consists of two parts:

$$
n=N_{r}+N_{i}
$$

, where $N_{\mathrm{r}}$ is the real part and $N_{\mathrm{i}}$ is the imaginary part. When electromagnetic wave propagates in absorptive medium the amplitude of the electromagnetic field decreases. This decrease is defined by following factor:

$$
\exp \left(-\frac{2 \pi N_{i}}{\lambda} \cdot l\right)
$$

, where $\lambda$ is the wavelength, and $l$ is the distance that optical wave passed in absorptive medium [8]. When the value of the wavelength is deep in the near infrared (IR) zone of the spectrum the value of the imaginary part of the effective refractive index increases, therefore the influence of the absorption should decrease [9].

For taking the absorption into account the new version of the modified beam-splitting (MBS-1) algorithm [10] was developed. It calculates light scattering matrices within geometrical and physical optics approximation for particles with flat facets. In addition, there is the feature that allows defining an arbitrary shaped particle in the new version of the algorithm.

\section{ESTIMATION OF ABSORPTION}

For estimation of the absorption influence to the light scattering process on ice particles with different shapes it was performed the calculation of light scattering matrices for the particles with two shapes: solid hexagonal column and arbitrary shaped particle (Figure 1). The calculation was performed with following parameters: random spatial orientation of particle, three different wavelengths with corresponded complex refractive indices (Table 1), and the maximal dimension of the particle varies from $10 \mu \mathrm{m}$ to $1000 \mu \mathrm{m}$. The aspect ratio of hexagonal column is 
corresponded to D. Mitchel dependency [11]. The size of the arbitrary formed particle varies proportionally. As the maximal dimension it means the distance between two the most distant vertices of the particle.
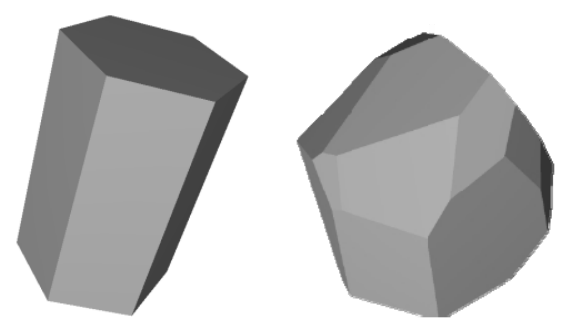

Figure 1 Particles for the calculation: the solid hexagonal column (left) and the arbitrary formed particle (right).

Table 1 Refractive indices (n) for chosen wavelengths $(\lambda)$

\begin{tabular}{|l|l|}
\hline$\lambda, \mu \mathrm{m}$ & $n$ \\
\hline 0.355 & $1.3243+i 3.6595 \cdot 10^{-9}$ \\
\hline 0.532 & $1.3116+i 2.6180 \cdot 10^{-9}$ \\
\hline 1.064 & $1.3004+i 1.9000 \cdot 10^{-6}$ \\
\hline 1.600 & $1.2893+i 3.5365 \cdot 10^{-4}$ \\
\hline
\end{tabular}

It is convenient to define the estimation quantity of the absorption influence in the backscattering direction as $\chi$ and for the whole diagram as $\psi$ :

$$
\begin{gathered}
\chi=\frac{M_{11}\left(180^{\circ}\right)-\bar{M}_{11}\left(180^{\circ}\right)}{M_{11}\left(180^{\circ}\right)} \cdot 100 \% \\
\psi=\frac{\int_{0}^{\pi} \bar{M}_{11}(\theta) \sin (\theta) d \theta}{\int_{0}^{\pi} M_{11}(\theta) \sin (\theta) d \theta}
\end{gathered}
$$

, where $M_{11}$ is the intensity of the scattering light without absorption, $\bar{M}_{11}$ is the same quantity with absorption.

\section{CALCUlation}

For laser sounding problems only backscattering point $\left(180^{\circ}\right)$ is interesting, but the whole scattering diagram is important for radiation transfer problems. The dependency of the maximal dimension of the particle $\left(D_{\max }\right)$ on $\chi$ in the backscattering direction for the solid hexagonal column and the arbitrary formed particle is presented in Figure 2 and the dependency of $D_{\max }$ on $\psi$ is in Figire 3a and 3b.



Figure 2 Dependence of $D_{\max }$ on $\chi$ for the solid hexagonal column and the arbitrary formed particle

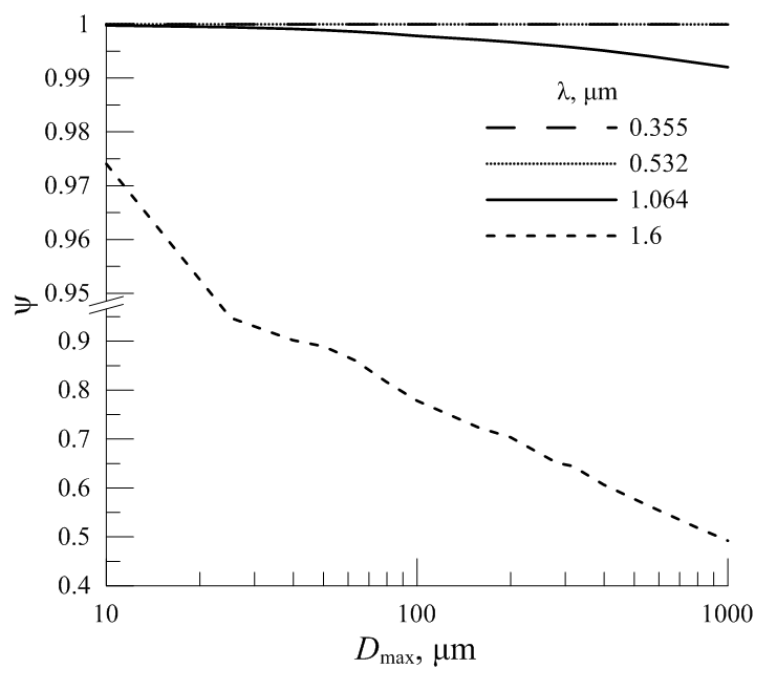

Figure 3(a) Dependence of $D_{\max }$ on $\psi$ for the solid hexagonal column 


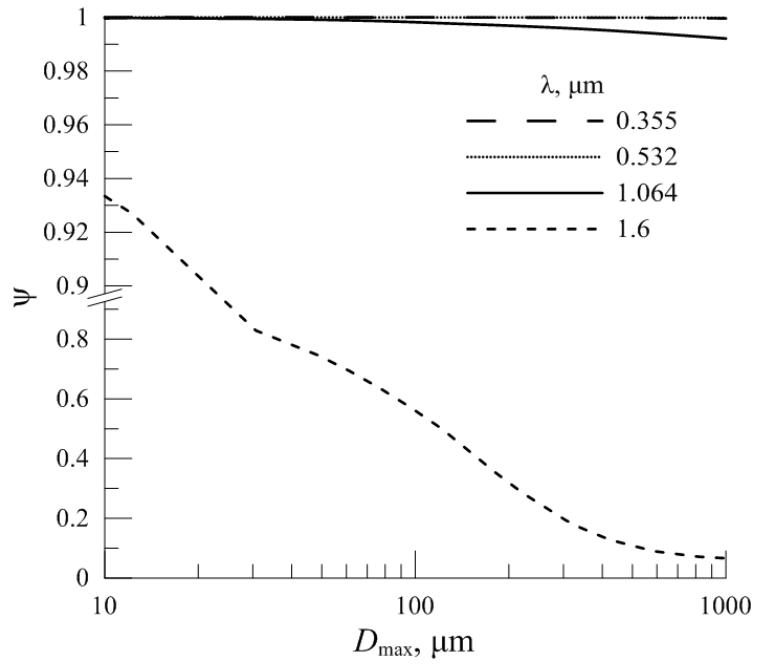

Figure 3(b) Dependence of $D_{\max }$ on $\psi$ for the arbitrary formed particle

\section{CONCLUSIONS}

The analysis of results show that the absorption influence is insignificant for the ultraviolet and visible zone of the spectrum $(0.355 \mu \mathrm{m}$ and 0.532 $\mu \mathrm{m}$ wavelengths) for solving of the light scattering problem for ice crystal particles of cirrus clouds, because it's value is less than basis points of percent regardless to the shape, orientation, and size of particle. However in the near IR zone the absorption influence could reach several dozen percent almost in the whole range of the shape and size of particles. It is convenient to choose $1.064 \mu \mathrm{m}$ with the value of imaginary part of the refraction index $\left(N_{\mathrm{i}}\right) 1.9 \cdot 10^{-6}$ as the threshold value of the wavelength where the absorption is significant. Thus for the wavelengths with $N_{\mathrm{i}}$ less than $1.9 \cdot 10^{-6}$ (near IR zone) it is obligatory to take the absorption into account for solving the direct light scattering problem. Moreover the absorption influence significantly depends on shape and size of particle for the $1.064 \mu \mathrm{m}$ wavelength. In conclusion, the absorption influence is less than $2 \%$ for the chosen range of size for hexagonal ice particles.

\section{ACKNOWLEDGEMENTS}

This work is supported by the Russian Foundation for Basic Research under Grants no. 18-05-00568, no. 18-55-53046, and no. 19-01-00351 and supported in part by Russian Science Foundation (18-77-10035).

\section{REFERENCES}

[1] Liou K.N. Influence of cirrus clouds on weather and climate processes: A global perspective // Mon. Wea. Rev. 1986. V. 114, N 6. P. 1167-1199.

[2] Kunz K.S., Luebbers R.J. Finite Difference Time Domain Method for Electromagnetics. - Boca Raton, FL: CRC Press, 1993

[3] Taflove A. Advances in Computational Electrodynamics: The Finite-Difference Time-Domain Method. - Boston: Artech House, 1998

[4] Purcell E.M., Pennypacker C.R. Scattering and absorption of light by nonspherical dielectric grains // Astrophys. J. — 1973. — T. 186. - C. 705-714.

[5] Yurkin M.A., Maltsev V.P., Hoekstra A.G. The discrete dipole approximation for simulation of light scattering by particles much larger than the wavelength // J. Quant. Spectrosc. Radiat. Transfer. - 2007. - T. 106. - P. 546-557.

[6] Yurkin M.A., Hoekstra A.G. The discrete-dipoleapproximation code ADDA: Capabilities and known limitations // J. Quant. Spectrosc. Radiat. Transfer. 2011. - T. 112. - P. 2234-2247.

[7] Volkovitcky O.A., Pavlova L.N., Petrushin A.G. Optical properties of the Ice Clouds. - Leningrad: Gidrometeoizdat, 1984. — P. 198.

[8] Bohren C.F., Huffman D.R. Absorption and Scattering of Light by Small Particles. - John Wiley \& Sons, 1986. - P. 660.

[9] Timofeev D.N., Konoshonkin A.V., Kustova N.V., Borovoi A.G. Backscattering matrices calculation for atmospheric ice crystals within the physical optics approximation with absorption effect // Proc. SPIE. 2018. V. 10833. 10833-174.

[10] Konoshonkin, A., Kustova, N., and Borovoi, A., "Beam splitting algorithm for the problem of light scattering by atmospheric ice crystals. Part 2 . comparison with the ray tracing algorithm," Atmospheric and Oceanic Optics, 28, 448-454 (2015). [11] Mitchell D.L., Arnott W.P. A model predicting the evolution of ice particle size spectra and radiative properties of cirrus clouds. Part II. Radiation. // J. Atmos. Sci. 1994. V. 51. P. 817-832. 